Die folgende Tabelle enthält eine Uebersicht der Mesitilolreihe.

$$
\begin{aligned}
& \text { Mesitilol . . . . } \mathrm{C}_{18} \mathrm{H}_{12} \\
& \text { Trichlormesililol } . \mathrm{C}_{18}\left\{\begin{array}{l}
\mathrm{H}_{9} \\
\mathrm{Cl}_{3}
\end{array}\right\} \\
& \text { Tribrommesitilol . } \mathrm{C}_{18}\left\{\begin{array}{l}
\mathrm{H}_{9} \\
\mathrm{Br}_{3}
\end{array}\right\} \\
& \text { Nitromesitilol . . . } \mathrm{C}_{14}\left\{\begin{array}{l}
\mathrm{H}_{12} \\
\mathrm{NO}_{4}
\end{array}\right\}(?) \\
& \text { Dinilromesililol . } \cdot \mathrm{C}_{18}\left\{\begin{array}{c}
\mathrm{H}_{10} \\
2 \mathrm{NO}_{4}
\end{array}\right\} \\
& \text { Trinitromesitilol . } \cdot \mathrm{C}_{18}\left\{3_{4} \mathrm{NO}_{4}\right\} \\
& \text { Mesililolschwefels. } \mathrm{HSO}_{4}, \mathrm{C}_{18}\left\{\begin{array}{l}
\mathrm{H}_{\mathrm{H}^{1}} \\
\mathrm{SO}_{2}
\end{array}\right\} \\
& \text { Nitromesidin (Maule) } \mathrm{C}_{18}\left\{\begin{array}{l}
\mathrm{H}_{1} \\
\mathrm{NI}_{2}^{1} \\
\mathrm{NO}_{4}
\end{array}\right\}
\end{aligned}
$$

Ueber das Nitromesidin, eine neue organische Base; von Georg Maule, Schüler des Royal College of Chemistry.

Die neuen Untersuchungen über die Zusammensetzung des Mesitilols, welche die merkwürdige Isomeric dieses Körpers mit dem Cumol, dem Liollenwasserstoff der Cuminsäurereihe, darthun, machten es wünschenswerth, einige weitere von ihm abgeleitete Verbindungen näher kennen zu lernen, um fernere Gewifsheit über die Richtigkeit dieser neuen Formel zu erhallen.

Es schien zu diesem Zweck nichts geeigneter zu seyn, als das Verhalten seiner Nitroverbindungen gegen Reduc- 
tionsmittel zu untersuchen. Die Bildung neuer, den Nitroverbindungen des Mesitilols entsprechender Alkaloide, die zu erwarten stand, und die genauen Melhoden, welche wir für die Aequivalentbeslimmung solcher Körper besitzen, versprachen eine zur Controle der Richtigkeit der verbesserten Formel besonders geeignete Reihe von Thatsachen darzubieten. Wenn man Cumol mit Salpetersäure behandelt, so erhält man zwei neue Körper, Nitro- und Dinitrocuinol, welche beide durch die Einwirkung von Schwefelwasserstoff in basische Verlindungen verwandelt werden. Der erste giebt das von Nicholson untersuchte Cumidin, während der zweite in dic von Cahours vor kurzem unler dem Namen von Nitrocumidin beschriebene Substanz verwandelt wird.

Da die Existenz eines mil Nitrocumol isomeren Körpers in der Mesitilolreihe immer noch zweifelhaft war, so schlug mir Dr. Hofmann vor, eine dem Nitrocumidin in ihrer $\mathbf{Z u}$ sammensetzung entsprechende Base darzustellen.

Die folgende Abhandlung enthält eine Beschreibung dieses newen Körpers, der wirklich ohne Schwierigkeit erhalten wird, und für den ich stalt der Bezeichnung Nitromesitilidin, welche ihm der Analogic nach zukäme, den Namen Nitromesidin vorschlage.

Ueberlälst man eine alkoholische Lösung von Dinitrocumol

$$
\mathrm{C}_{18}\left\{\begin{array}{l}
\mathrm{H}_{10} \\
\mathrm{NO}_{4}
\end{array}\right\}
$$

der Einwirkung von Schwefelwasserstoff, so nimmt die Fliissigkeit eine dunkle Farbe an und setzt nach und nach eine grofse Menge Schwefel ab, während der Geruch nach Schwefelwasserstoff verschwindet. Fügt man Chlorwasserstoffsäure zu, so schlägt sich abermals Schwefel nieder, und man erhält nach dem Filtriren eine klare Flüssigkeit, welche mit einer Lösung von Kali oder Ammoniak vermischt, einen reichlichen gelben 
Niederschlag giebt. Dieser Niederschlag ist Nitromesidin in noch unreinem Zustand.

Um dasselbe rein zu erhalten, wird es wiederholt in Chlorwasserstoffsäure gelüst und durch ein Alkali wieder gefält. Auf diese Art werden kleine Mengen von immer noch anhängendem Schwefel abgeschieden und die Substanz nimnt allmälig ein glänzend gelbe Farbe an. Ein - oder zweimaliges Krystallisirenlassen aus Alkohol genügt jetzt, um dieselbe vollkommen rein zu erhallen.

Zusammensetzung des Nitromesidins. - Die folgenden Analysen wurden mit $z u$ verschiedenen Zeiten dargestellten und bei $100^{\circ}$ getrockneten Krystallisationen von reinem Nitromesidin angestellt.

I. 0,2861 Grm. Substanz gaben 0,6309 Grm. Kohlensäure und 0,1716 Grm. Wasser.

II. 0,2072 Grm. Substanz gaben 0,4561 Grm. Kohlensäure und 0,1264 Grm. Wasser.

III. 0,2264 Grm. Substanz gaben $0,4967 \mathrm{Grm}$. Kohlensäure und $0,1387 \mathrm{Grm}$. Wasser.

In 100 Theilen :

\begin{tabular}{lrrr} 
& \multicolumn{1}{c}{ I. } & \multicolumn{1}{c}{ II. } & \multicolumn{1}{c}{ III. } \\
Kohlenstoff & $\mathbf{6 0 , 1 4}$ & $\mathbf{6 0 , 0 3}$ & $\mathbf{5 9 , 8 5}$ \\
Wasserstoff & 6,66 & 6,77 & $6,80$.
\end{tabular}

Eine Bestimmung des Stickstoffs nach der Methode von Bunsen gab folgende Resultate :

Stand in Corrig. Stand des Queck- Temp. Bar, Vol. b. $1^{\mathrm{mm}}$ d. Rühro Vol. silbers in d. Wanne Druck u. $0^{\circ} \mathrm{C}$.

Kohlensäure

u. Stickstoff,

feucht . . $137 \quad 136,3 \quad 249,5 \quad 9,0 \quad 779,1^{\mathrm{mm}} 89,6606$

Trockner

Stickstoff . 19,4 $\quad 17,2 \quad 250,0 \quad 9,5 \quad 773,5^{\mathrm{mm}} \quad 9,362$.

Verhältnifs der Kohlensäure- und Stickstoffäquivalente :

$$
89,6606-9,362: 9,362=100: 11,6 \text {. }
$$


Nimmt man den millleren Werlh $(60,01)$ der gefundenen Kohlenstoffprocente, so ergielt die Berechnung $16,31 \mathrm{pC}$. Slicksloff:

Obgleich dieses Resultat nicht so genau ist, als die, welche man gewölntich erhält, so erwähne ich es doch als eine $\mathrm{Bc}-$ stäligung der aus den obigen Analysen abgeleitelen Formel :

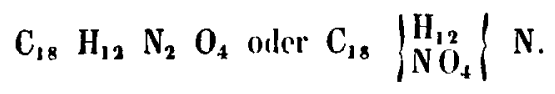

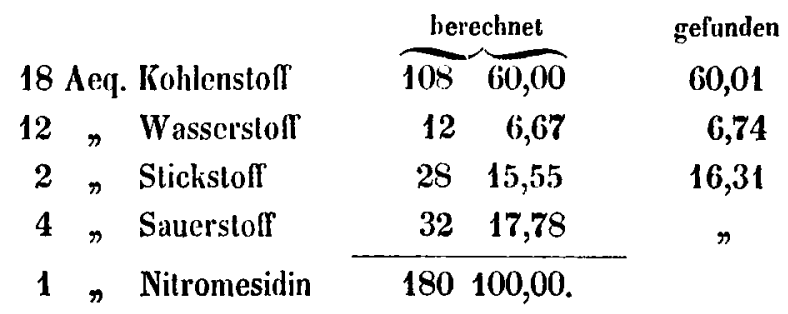

Eigenschaften des Nitromesidins. - Man crhält diesen Körper rein in langen nadelförmigen Krystallen von goldgelber Farbe. Die Krystalle werden schon unter $100^{\circ}$ flüssig und erstarren beim Kaltwerden zu ciner Masse von strahlenförmig angeordneten Nadeln. Sie sind sehr lïslich in Alkohol und Aether und in geringem Grade in Wasser, dem sie eine blafsgelbe Farbe ertheilen. Das Nitromesidin ist bei $100^{\circ}$ ohne Zerselzung flüchtig und sein Dampf brennt mit blauer Flaınme. Seine Lösungen sind neutral und haben einen unangenehmen biltern Geschmack.

Verbindungen des Nitromesidins. - Das Nitromesidin löst sich leicht in Säuren und bildet krystallinische Salze mit denselben. Seine basischen Eigenschaften sind indessen sehr schwach und die meisten seiner Salze verändern sich leicht. Alle dicjenigen, die ich erhielt, zerselzten sich, mit Ausnahme des Platindoppelsalzes und des phosphorsauren Salzes, schon bei der blofsen Berührung mit Wasser. Sie sind löslich in Alkohol und ihre Lösungen haben eine saure Reaction. 
Chlorwousserstoffsaures Nitromesidin. - Zur Darstellung dieses Salzes wird die Base in verdünnter Salzsäure gelöst und dic Lösung verdampft. Lälst man das Ganze nun stehen, so krystallisirt das Salz in farblosen Nadeln. Da dieses Salz durch Wasser zerselat wird, so wurde die Lösung auf dem Wasserbade zur 'Trockne verdampft, bis alle freie Säure weggregangen war. Bei $100^{\circ}$ getrocknet gaben $0,3148 \mathrm{Grm}$. des Salzes 0,5716 Kohlensäure und 0,1703 Wasser.

0,1593 Grm. des Salzes gaben 0,1051 Chlorsilber.

Diese Zahlen entsprechen der Formel :

$$
\begin{array}{llllll}
\mathrm{C}_{18} & \mathrm{H}_{12} & \mathrm{~N}_{2} & \mathrm{O}_{4}, \mathrm{H} & \mathrm{Cl} .
\end{array}
$$

18 Aeq. Kohlenstoff

13 Wasserstoff

2 Stickstoff

4 \Sauerstoff

1 , Chlor

1 Chlorwasserstoffs. Salz $216,5 \quad 100,00$.

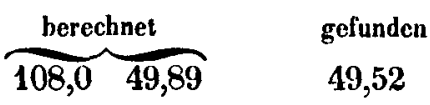

$13,0 \quad 6,00 \quad 6,01$

$28,0 \quad 12,93$

$32,0 \quad 14,78$

$35,5 \quad 16,40$

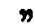

》

16,32

Nitromesidin-Platinchlorid. - Setzt man Platinchlorid in Uelerschufs zu einer gesälligten Lösung des salzsauren Salzes, so fällt bei der Abkühlung das Doppelsalz von Nitromesidin und Platinchlorid in Gruppen von gelben Krystallen aus der Lösung. Un das Salz von einem Ueberschuls von Platinchlorid zu befreien, wurde es mit Wasser gewaschen und dann aus Alkohol krystallisiren lassen. Um die Formel $\mathrm{zu}$ controliren und das Atomgewicht der Substanz zu bestimmen, wurden vier sorgfältige Platinbestimmungen gemacht und für jede derselben das Salz besonders dargestellt.

l. 0,2122 Grm. Platinsalz gaben 0,0544 Platin.

\begin{tabular}{|c|c|c|c|c|}
\hline II. 0,2587 & " & \# & n & 0,0661 \\
\hline III. 0,1625 & 》 & $\pi$ & r & 0,0410 \\
\hline IV. 0,1806 & $\eta$ & » & 》 & 0,0462 \\
\hline
\end{tabular}


V. $0,03358 \mathrm{Grm}$. Platinsulz gaben 0,3470 Kohlensäure u. 0,1083 Wasser.

VI. $0,3741 \quad \gg \quad \gg \quad$ $\quad 0,3838 \quad \gg \quad, 0,1167$ Wasser.

Diese Zahlen entsprechen der Formel :

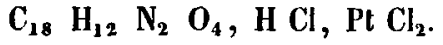

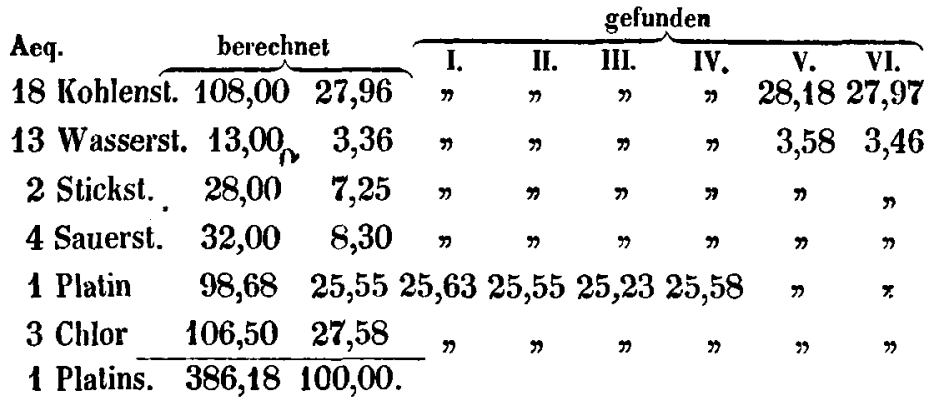

Schwefelsaures Nitromesidin. - Löst man die Basis in siedender verdünnter Schwefelsäure, so schlagen sich beim $A b-$ kühlen weifse seidenglänzende Krystalle des schwefelsauren Salzes nieder. Es wird durch Wasser zersetzt, indem sich die Base abscheidet. Die Analyse wurde daher nicht gemacht.

Salpetersaures Nitromesidin wird gebildet, wenn man Nitromesidin in verdünnter Salpelersäure lóst. Wird die Lösung mit einem Ueberschufs an freier Salpetersäure verdampft, so zersetzt sie sich bei einem gewissen Grade der Concentration, indem sich rothe salpetrigsaure Dämpfe entwickeln und als Product der Zersetzung eine rothe ölige Substanz zurückbleibt.

Dreibasisches phosphorsaures Nitromesidin. - Wenn die Basis in einer Lösung von Phosphorsäure aufgelöst wird, so krystallisirt das Salz in blältrigen Krystallen von schön orangegelber Farbe. Nach Entfernung der freien Säure bleibt das Salz vollkommen rein zurück. Es wurde bei $100^{\circ}$ getrocknet und dann analysirt.

J. $0,2471 \mathrm{Grm}$. des Salzes gaben $0,4580^{\circ}$ Kohlensäure und 0,1413 Wasser. 
II. 0,3058 Grm. des Salzes gaben 0,0529 phosphors. Billererde. Diese Zahlen entsprechen der Formel :

$$
3\left(\begin{array}{lllll}
\mathrm{H} \mathrm{C}_{18} & \mathrm{H}_{12} & \mathrm{~N}_{2} & \mathrm{O}_{4}
\end{array}\right), \mathrm{PO}_{8} \text {. }
$$

54 A eq. Kohlenstoff

39 Wasserstoff

6 Stickstoff

20 , Sauerstoff

1 》Phosphorsäure

1 "Phosphors. Salz berechnet

$\overbrace{324,0}^{50,70}$

$39,0 \quad 6,10$

$84,0 \quad 13,14$

$160,025,06$

$32,0 \quad 5,00$ gefunden

50,54

6,35

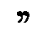

"

4,86

Wenn man einen grofsen Ueberschufs an Thosphorsäure nimmt, so erhält man ein saures Salz, welches nur ein Aequivalent Nitromesidin zu enthalten scheint.

Zersetzungsproducte des Nitromesidins. Die geringe Menge von Substanz, die ich zur Verfügung haile und das langwierige Verfahren, um das Nitromesidin zu erhalten, verhinderten mich, seine Zersetzungsproducte in bedeutenderer Ausdehnung zu studiren.

Einwirkung des Broms und Chlors auf Nitromesidin. Bringt man Nitromesidin mit Brom in Berührung, so zeigt sich eine heftige Reaction und es entsteht eine dunkele ölige Substanz. Es ist diefs ein merkwürdiger Uebergang, da er uns den Unterschied zwischen dem Nitromesidin und der mit demselben isomeren Verbindung, dem Nitrocumidin, zeigt; wenn dieses letztere nämlich mit Brom behandelt wird, so erbält man einen festen krystallinischen Körper.

Behandelt man eine alkoholische Lösung von Nitromesidin mit Chlor, so bekommt man eine fleischfarbene feste Masse, welche sich in siedendem Aether löst und in der Kälte wieder daraus niederfällt.

Die Reduction des Trinitromesitilols mit Schwefelwasserstoff schien uns sehr bemerkenswerth, da bis jelzt keine Nitrosäureverbindung mil 3 Alomen Lntersalpetersäure dieser Be- 
handlung unterworfen wurde. Das Trinitromesitilol ist sehr schwer zu reduciren; ich liefs Schwefelwasserstoff auf eine beträchlliche Menge dieser Substanz einige Wochen lang einwirken und fand an Ende dieser Zeit, dafs nur eine sehr kleine Menge verändert war. Ich erhielt indessen eine hinreichende Menge des Productes, um die Existenz ciner basischen, aus dem Nitromesitilol entstandenen Verbindung festzustellen, obgleich mich der geringe Betrag dieser Substanz, und die Schwierigkcit sic zu reinigen, dazu bewog, ihre Untersuchung auf eine spätere Zeit aufzusparen.

\section{Untersuchung über die chemische Natur des Wachses;} von Benjamin Collins Brodie.

\section{Ueber das Myricin*).}

Zwischen die Untersuchung der Cerolinsäure und diejenige des Rückstandes, der nach der Abscheidung dieser Säure von dem Bienenwachs zurückbleibt, habe ich die Untersuchung des chinesischen Wachses eingeschaltet. Bei der Verseifung des chinesischen Wachses erhalten wir, wie ich gezeigt habe, eine mit der Cerotinsäure des Bienenwachses identische Säure nebst dem Alkohol derselben, so dafs die chemische Entstehungsgeschichte dieser Substanzen in genauem Zusammenhang steht. In dem chinesischen Wachs finden wir aulserdem eine Substanz, die in der

*) Siehe diese Annalon Bd, LXVII, S. 180. 